\title{
DISORDERS OF OCULOMOTOR FUNCTION IN LESIONS OF THE OCCIPITAL LOBE*
}

\author{
BY
}

\author{
MORRIS B. BENDER, DONALD M. POSTEL, and HOWARD P. KRIEGER
}

\begin{abstract}
From the Departments of Neurology of New York University, Bellevue Medical Centre, and the Mount Sinai Hospital, New York City
\end{abstract}

The physician and even the neurologist associates the occipital lobe with visual functions. Rarely does one attribute motor disturbance to lesions in this region of the brain. While there is no doubt that normal vision is dependent on intactness of the calcarine cortex and the subcortical optic radiations, there is apparently little clinical evidence to indicate that the occipital area plays a role in the movements of the eyes. From experiments in animals and cortical stimulations in man it is apparent that the occipital lobe must have an influence on eye movements.

In monkeys various types of eye movements have been elicited from the occipital cortex and subcortex (Shäfer, 1888; Luciani and Tamburini, 1879; Grünbaum and Sherrington, 1901; Walker and Weaver, 1940; Crosby and Henderson, 1948; Lilly, Hughes, and Galkin, 1956; Krieger, Wagman, and Bender, 1955). While these effects are easily obtained in the alert and less readily in the anaesthetized monkey, ocular deviations are difficult to elicit on electric stimulation of the occipital cortex in man. Walker and Weaver (1940) stated that eye movements can be elicited from the occipital area in all animals but man. Foerster (1931), on the other hand, stated that ocular deviations could be evoked on stimulation of area 19 but not areas 18 or 17. Penfield and Boldrey (1937) reported two cases with contralateral ocular deviation after stimulation of area 19. However, in 1950 Penfield and Rasmussen stated that in 31 patients 48 loci for eyeball movement were found and "all were located rostral to the central sulcus". To explain the previous observations the following footnote is then added: "In earlier operations in which less physiological stimulating currents were used, eye turning was elicited in one patient from the temporal lobe and in three patients from the

* This work was aided in part by grants No. B-174 (S.D.) and No. B-294(C) from the United States Public Health Service. parietal lobe". The latter three patients include the two in whom eye movements were elicited from area 19. Penfield similarly accounted for the difference between his and Foerster's results by noting that the latter used much stronger stimuli and evoked adversive convulsions including the eyes. In their work Penfield and his associates (Penfield and Boldrey, 1937; Penfield and Rasmussen, 1950) did not consider any epileptiform reactions as positive results. However, in recent work on the monkey with implanted electrodes Lilly and his colleagues (1956) have evoked adversion, including the eyes, upon stimulation of the lateral surface of the occipital lobe and not isolated eye movements.

While there are examples of excitatory influences on eye movements produced by stimulating the occipital lobe, there is very little information on oculomotor deficits as a result of lesions in this area. Gordon Holmes (1921) described defects in visual fixation and fusion movements of the eyes in gunshot wounds of the occipital lobes. The so-called voluntary movements which remain are not perfect. For example, fixation other than straight forward cannot easily be maintained and may be accompanied by nystagmus. These abnormalities are found even though there is no paralysis of the individual eye muscles involved. Thus it is reflex maintenance of conjugate gaze in any one direction or even centering of eyes which is defective and the chief complaint is usually blurred vision, which increases with any movement of the line of vision. This blurred vision persists with one eye closed, thereby differentiating it from diplopia. Although bilateral occipital lobe damage is usually necessary for this syndrome, reflex maintenance of gaze may be defective unilaterally. When it is bilateral it becomes even more marked. The post-mortem examinations of cases by Gordon Holmes (1921) revealed that this defect in fixation was related to "interruption of the cortico-tectal fibres which form the efferent link 
of the reflex arch; the disease involved the dorsal portion of the thalamus including the pulvinar". In addition, Gordon Holmes described occipital lesions as producing paresis of gaze to the side opposite the lesion. In his cases this occurred only in hemianopics who were mentally obtunded, suggesting that what Holmes may have observed is what has been described as hemispatial inattention or hemispatial agnosia. Dejerine and Roussy (1905) observed such paresis after a recent occipital injury in a previously blind person, suggesting that vision is not the factor in these cases. Gordon Holmes (1921) also noted that many hemianopics could not accurately fixate targets on the hemianopic side, despite preservation of macular vision and accurate fixation of a target in the midline. Holmes concluded that such a defect could not be due to visual loss but was due to unilateral loss of the visual reflexes needed for maintaining the position of the eyes achieved by voluntary ocular movement.

In contrast to these observations and deductions are those of Penfield and Rasmussen (1950). They amputated one occipital lobe and found the only defect to be a contralateral homonymous hemianopia. There was no difficulty in oculomotor functions. Excision of the occipital cortex outside the calcarine region produced no defect in vision or oculomotor function of which the patient was aware. Destruction of the occipital lobe in monkeys leads to contralateral hemianopia and at most to a transient defect in contralateral conjugate gaze which is associated with turning of the entire body about its long axis in the direction of the side of the lesion.

The present study concerns alterations in oculomotor activity appearing subsequent to lesions of the occipital lobes in two patients.
Case 1.-D. G. developed hypertension (160-170/ $100-110 \mathrm{~mm} . \mathrm{Hg}$ ) at the age of 33 . At 37 years of age he suffered, in rapid succession, a coronary occlusion, a popliteal thrombus, multiple pulmonary infarcts, and an embolus to the right posterior cerebral artery. The latter was followed by an almost congruent left homonymous hemianopia, spots before the eyes, and blurred vision. In 1949 , at the age of 41 , he was completely blind for one hour. This was followed by left-sided blindness and the subjective experience of being dragged to the left by the head and eyes.

The patient was admitted to the Mount Sinai Hospital for special studies. The visual sensory symptoms and signs of this patient just after the episode of complete blindness have been reported previously (Bender and Kahn, 1949). Essentially, there was an incongruent left homonymous hemianopia with scotomas and a small relative scotoma in the right upper quadrants (Fig. 1). These defective fields were the site of trouble in perception of motion, form, distance, colour and afterimagery. Additional signs of neurological disease were hyperaesthesia of the left upper lip and right wrist. There was transient evidence of a hemiparesis affecting the right foot. Radiographs of the skull, the cerebrospinal fluid, and an electroencephalogram were normal. The diagnosis was bilateral and successive occlusion of the posterior cerebral arteries.

The most marked oculomotor defect found after the second occlusion was nystagmus on fixation of a target during deviation of the eyes to the side of the maximum $\triangle$ field defect (left). The nystagmus was a shimmering, cogwheel type of eye movement apparent on attempted fixation of a target at the near point. During fixation at a distance this nystagmus was absent. When present, at a near point, nystagmus was most marked in the fixating right eye, the non-fixating eye being turned inward, giving it the appearance of making a strong attempt at convergence. Nausea and vertigo developed when such fixation was prolonged.

Double vision was experienced during fixation at the near point or at a distance. If the patient looked at his

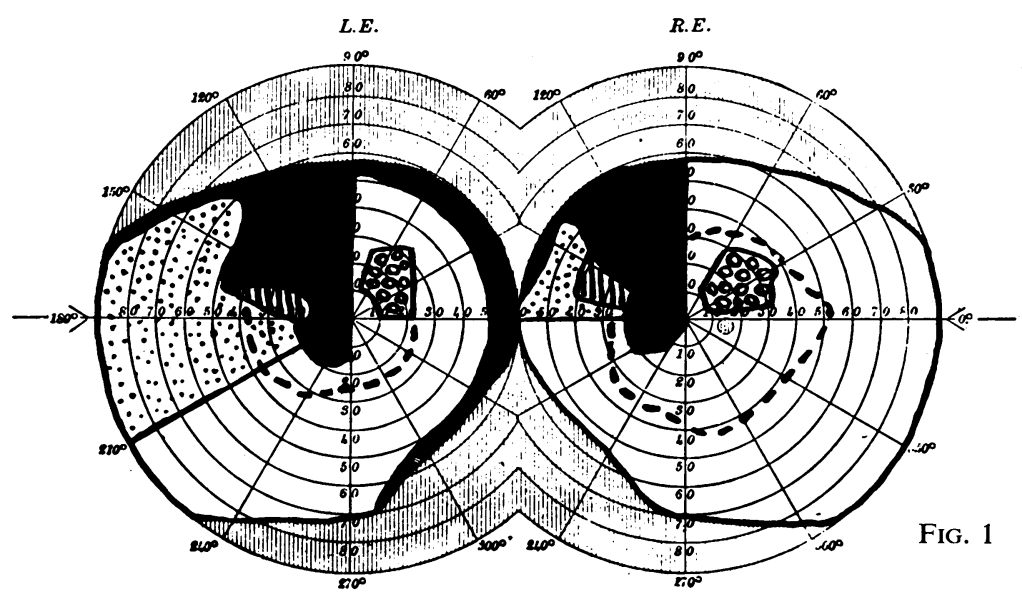

Fig. 1.-Perimetric and tangent (opposite page) screen visual fields of D. G. Illumination, daylight; $5 \mathrm{~mm}$. white target at $33 \mathrm{~cm}$. Solid black, absence of perception of motion. Vertical lines, movement perceived but marked blurring and fluctuation in appearance of target. Stippling, target less blurred. Small circles and horizontal dashes, blurred or grey vision. The dashes in the perimetric fields outline the field of vision for the colour red. 


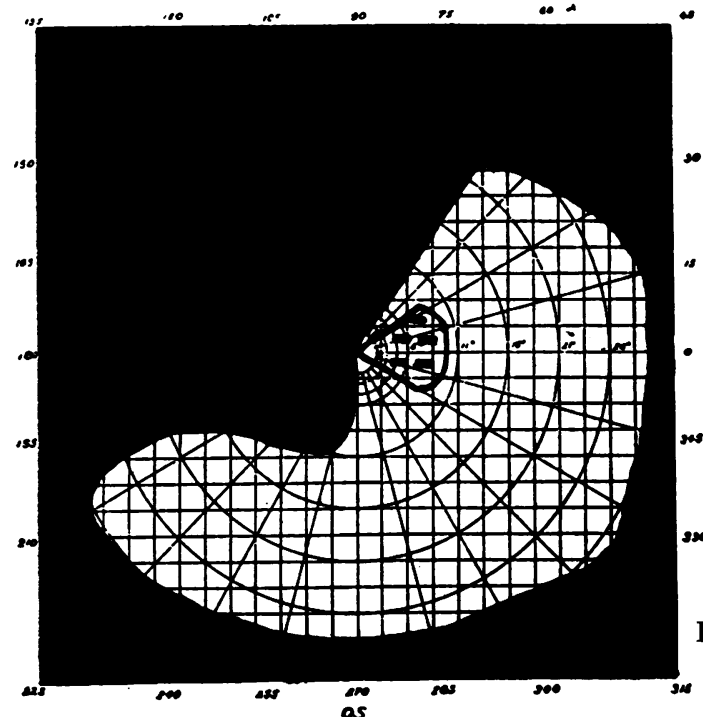

own finger he saw two parallel fingers one overlying the other. He called this experience " transparency" since each finger was seen in its entirety despite the overlap. The degree of image separation increased the farther the target was removed from the patient. This diplopia was best demonstrated by turning the head to the extreme left while the eyes were turned to the right and down, that is, toward the least defective field of vision. Under these conditions a target in the left or defective field was seen with an associated false image slightly higher as well as to the left of the real image. Marked bilateral nystagmus accompanied any attempt to fixate under these conditions. Concomitantly, the non-fixating eye converged.

Fifteen months after the onset of this oculomotor disturbance the patient was admitted to Bellevue Hospital. At this time a marked dissociated nystagmus was observed whenever the patient attempted to fix an object in his left field of vision. Shortly afterwards he died in cardiac failure.

Post-mortem examination was limited to the brain which showed infarction of the under surface of both occipital lobes (Figs. 2a and 2b). The infarct was largest on the right side extending forward but not into the
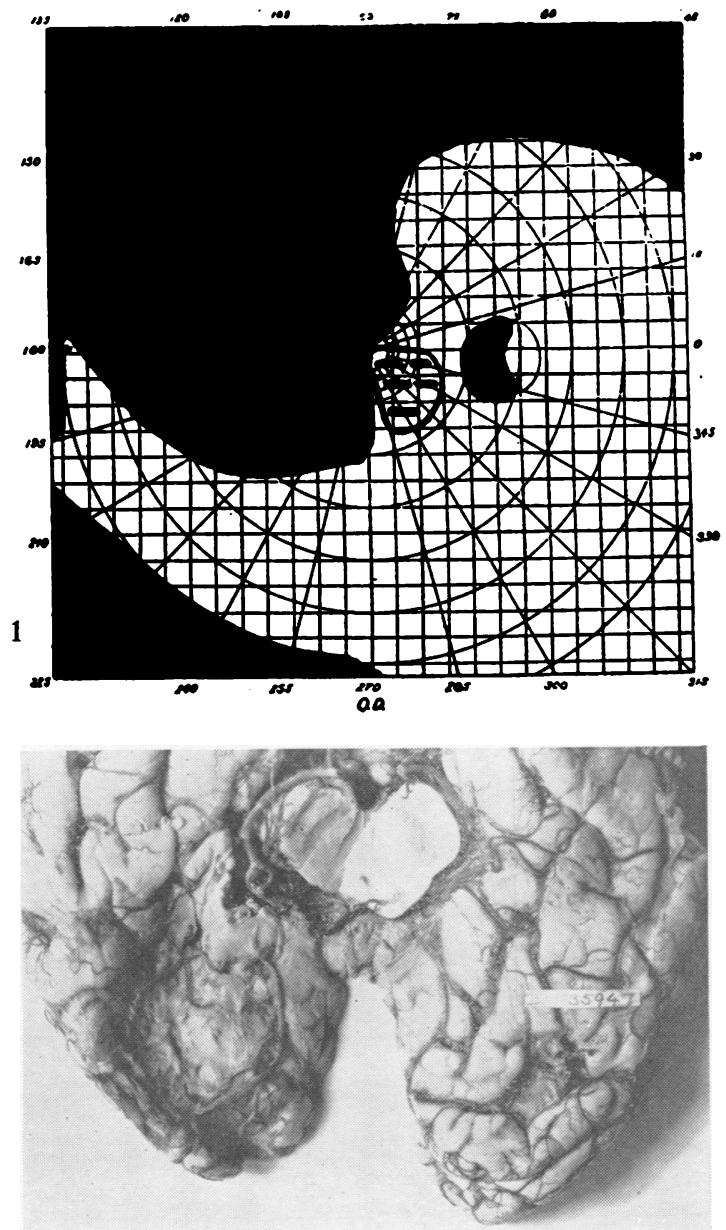

FIG. 2a.-Photograph of the inferior surface of the brain of $D$. G. The right occipital lobe is the site of an infarction throughout its inferior aspect. Most of the posterior part of the right lingual gyrus is missing exposing branches of the posterior cerebral artery lying within the calcarine and other fissures. The inferior surface of the remnants of the right cuneus is exposed. Most of the right occipital pole is destroyed. The inferior surface of the left occipital lobe is less severely damaged, the major destruction occurring in the lingual and fusiform gyri rostrally and thus affecting mainly the peristriate and parastriate areas.
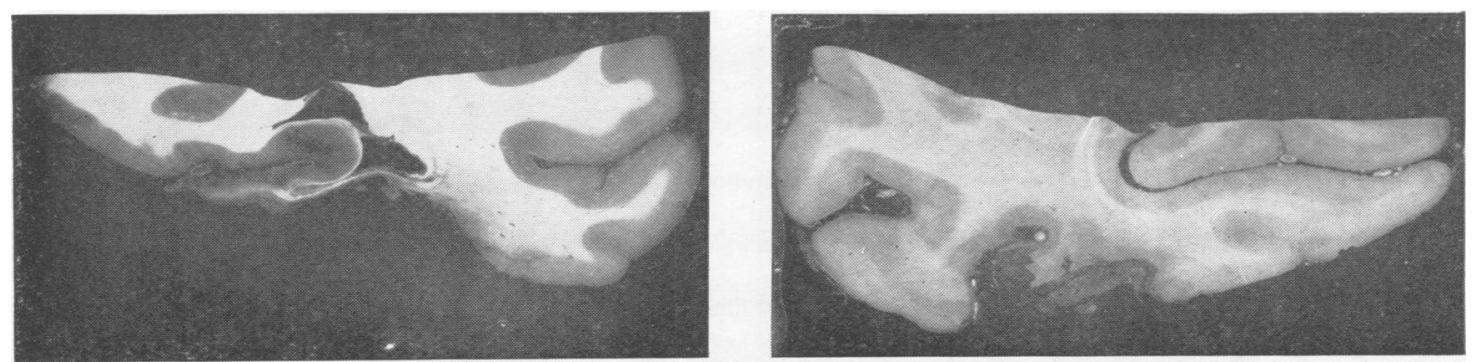

FIG. 2b.-Representative coronal sections through each occipital lobe demonstrating the depth of the lesion. The right lobe is more extensively involved. 
temporal lobe and including Brodman's area 18, that part of area 17 lying below the calcarine fissure, and all of the tip of the occipital lobe. The lesion on the left was limited to a small region mainly of area 18 . There were no lesions in the brain stem.

Case 2.-G. S. K., aged 57, first experienced a clonic, adversive seizure affecting the head and eyes at the age of 26. This patient was first studied by Foerster in $\mathbf{1 9 2 5}$ and 1926. Since then these attacks have recurred weekly and occasionally two or three times in one week. During the first two years consciousness was lost three times. The attacks have consisted of a sensation of something
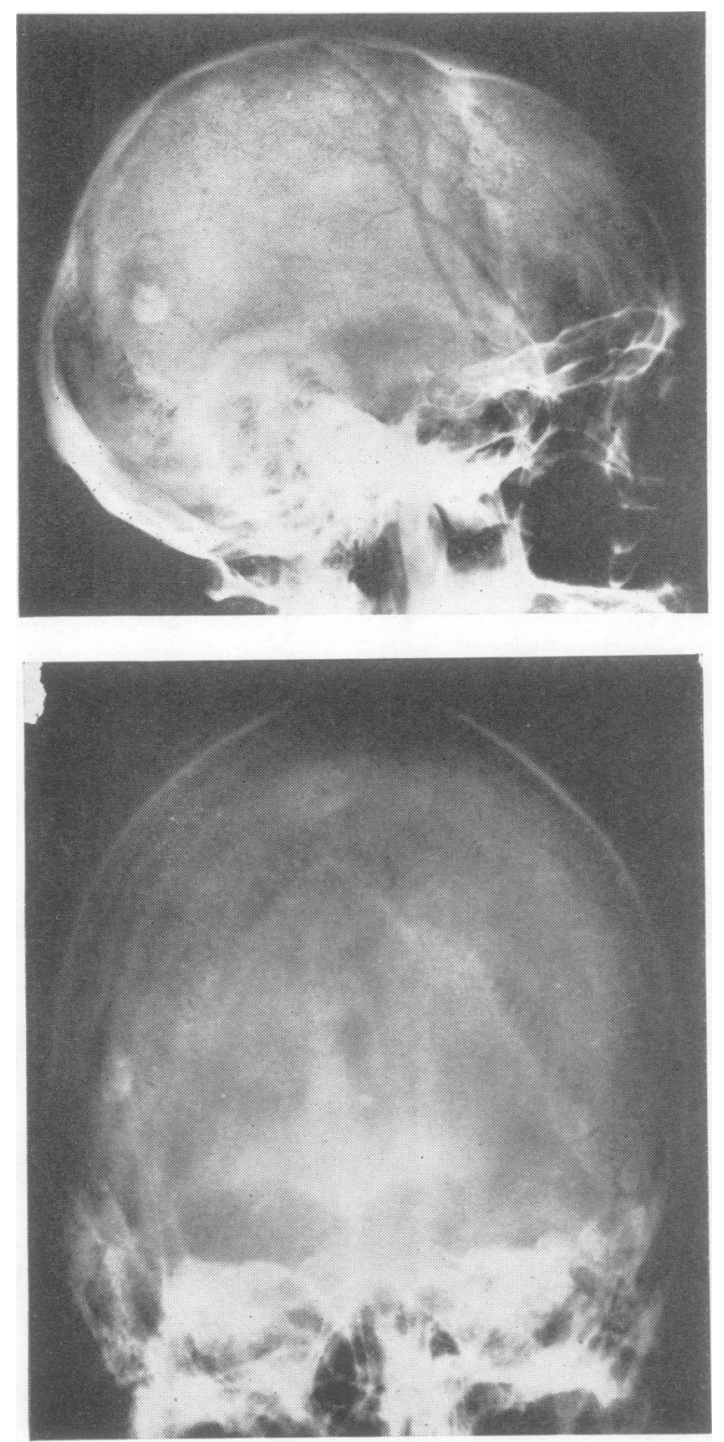

Fif. 3.-Lateral and anterior-posterior views of the skull of G. S. K. demonstrating the calcific lesion within the right occipital lobe. impending, followed by anxiety, then a sensation of $\mathrm{O}$ blood or warmth rushing to the head, and finally clonic jerking of the head and eyes to the left superimposed upon tonic adversion of these parts to the same side. Each attack lasted a few seconds. The tongue was not affected. Occasionally paraesthesias affecting the left lower extremity and possibly the left hand appeared after an attack. There was no diplopia, blurred vision, optic illusions, or. visual hallucinations. Four years before the first attack the patient was in a train accident. He suffered a blow to the head but did not lose consciousness. No other aetiological agent is known.

The patient was examined at the office of M. B. Bender on June 20,1947, and again in 1953. The neuro- $\bar{C}$ logical and mental states did not reveal any abnormality. 흘 An electroencephalogram was normal in 1947 and $\bar{\omega}$ again in 1956. Repeated radiological examinations of the skull have shown a right occipital calcified mass which has not grown in size (Fig. 3). It is assumed that the anatomical site of the lesion which causes the adversive $\overrightarrow{0}$ head and eye movements to the left is in the right occipital lobe and manifested by the calcification.

\section{Discussion}

In summary, two cases of disturbances in oculomotor function associated with lesions of the 0 occipital lobe have been described. One had is unilateral disease, the other bilateral sequentia $\vec{\omega}$ disease. The common symptom was abnormat repetitive eye movements. The case of unilateraf calcification in the occipital lobe had contraversive attacks, the other had nystagmus when attempting to fix in his most defective field of vision. It shoula be stressed that this patient had a visual field defeco.

These two cases thus support the concept that . in man the occipital lobe participates in oculomotor functions. This is in agreement with Foerster's (1931) observations and those of workers who have $\bar{\partial}$ experimented with animals. It may be asked whether such concepts as the visual fixation reflex and the $\stackrel{\square}{\unrhd}$ theory of balance between the forces effected through $\overrightarrow{\vec{F}}$ each occipital lobe may help in analyzing these two cases.

The nystagmus of the case with bilateral disease was apparently the type associated with defects in the postulated visual fixation reflex. It will be recalled that the nystagmus became most marked when the eyes were turned toward the most defective 3 visual field while the head was turned in the opposite direction. It is possible to agree with Holmes (1921) and say that this was nystagmus secondary to $a_{0}$ defective fixation reflex since vision was partly intact. However, it is not altogether clear why the $\frac{D}{O}$ position of the head should play a role in this symptom and why, as in the second case, it is $N$ possible to have attacks of clonic adversive eye $\mathrm{N}$ movements without evidence of a fixation deficit or visual experience. 
A definite answer cannot be given but some suggestions arise from known experimental data. Shäfer (1888) showed that the two cerebral hemispheres were in physiological balance as far as effecting forces which move the eyes in the horizontal plane, each hemisphere exciting contralateral conjugate deviation. Sherrington (1893) demonstrated that there were active inhibitory forces also involved in these horizontal conjugate movements. Bender (1955) postulated a third set of vectors, the centering forces or those which bring the eyes to the midposition and constantly counteract the deviation forces. If the existence of these forces is granted then it may be proposed that the altered oculomotor activity observed in the two cases under consideration had a common physiological mechanism, namely, imbalance among the generators of these forces, whether these generators lie in the anatomically diseased structures or some other place along the pathways which pass through these structures. Such an explanation does not invoke centres for specific types of horizontal or other eye movements and it poses the problem in a form allowing an experimental approach. Such a mechanism might also underlie the other oculomotor defects associated with the disease of the occipital lobes and commonly explained as loss of the "visual fixation reflex".

There are observations tending to support such a vector concept suggesting that all eye movements, normal and abnormal, are the result of forces generated at many places along the neuraxis, i.e., cerebrum, basal ganglia, brain-stem, cerebellum, vestibular nuclei, the various oculomotor nuclei and the retina, vestibular receptors and the proprioceptors of the head and neck (Bender, 1955; Spiegel and Sommer, 1944). Of particular interest here are some recent observations on the monkey. It was noted that nystagmus could be induced in a conscious monkey if the animal's attention were attracted ipsilaterally to an occipitally applied electric stimulus to the cortex. During such simultaneous stimulation there was nystagmus (Krieger et al., 1956). A possible interpretation of this nystagmus is that it is the result of a rivalry between the attention-engendered forces and the electricallyinduced forces. Nystagmus was also noted upon stimulation of various parts of the occipital and frontal eye fields without apparently requiring the factor of attention. No particular anatomical or physiological pattern was found for this nystagmus, suggesting that perhaps the effect of the added stimulus was to create an imbalance between the deviating and centering forces, which imbalance was noted as nystagmus.

Why this imbalance appears as periodic, clonic movements rather than tonic deviation cannot be said. To state that the eyes partake in convulsive phenomena is descriptively true but adds little to our understanding of the mechanism whereby the abnormal activity of the occipital lobe tissue results in repetitive patterned contractions of the eye muscles. Even if it were known how the calcified mass in Case 2 caused abnormal activity in the occipital lobe, the question of why this was manifest as clonic eye movements while in a deviated position still remains open. There is no satisfactory physiological explanation for the two oculomotor syndromes described in this paper. The occurrence of these eye disturbances supports the concept of occipital lobe influence on oculomotor activity. It is remarkable that so few cases of oculomotor deficit have been reported in disease of the occipital lobe. It would seem that this is an inadvertent error of omission brought about by a combination of the prominence of the sensory defect and the usual classification of the occipital lobe as a sensory organ.

\section{Summary}

Two cases of defects in oculomotor activity are described. One followed occlusion of both posterior cerebral arteries; the other was associated with a calcified mass in one occipital lobe.

The concept of the occipital oculomotor field is reviewed and the pertinent conflicting data are presented.

It is concluded that the occipital lobe influences the function of the oculomotor apparatus.

\section{REFERENCES}

Bender, M. B. (1955). A.M.A. Arch. Neurol. Psychiat., 73, 685. , and Kahn, R. L. (1949). Journal of Neurology, Neurosurgery, and Psychiatry, 12, 196.

Crosby, E. C. and Henderson, J. W. (1948). J. comp. Neurol., 88, 53. Dejerine, J., and Roussy, G. (1905). Rev. neurol. (Paris), 13, 161.

Foerster, O. (1931). Lancet, 2, 309.

Grünbaum, A. S. F., and Sherrington, C. S. (1901). Proc. roy. Soc., 69, 206.

Holmes, G. (1921). Brit. J. Ophthal., 5, 241.

Krieger, H. P., Wagman, I. H., and Bender, M. B. (1955). Trans. Amer. neurol. Ass., p. 209.

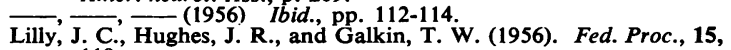
i19.

Luciani, L., and Tamburini, A. (1879). Rivista sperimentale di freniatria e di medicina legale, $5,1-76$.

Penfield, W., and Boldrey, E. (1937). Brain, 60, 389.

, and Rasmussen, T. B. (1950). The Cerebral Cortex of Man. Macmillan, New York.

Schäfer, E. A. (1888). Brain, 11, 145.

Schäfer, E. A. (1888). Brain, 11, 145.
Sherrington, C. S. (1893). Proc. roy. Soc., 53, 407.

Spiegel, E. A., and Sommer, I. (1944). Neurology of the Eye, Ear, Nose and Throat. Grune \& Stratton, New York.

Walker, A. E., and Weaver, T. A. Jr. (1940). J. Neurophysiol., 3, 353. 